Open Access

\title{
Intraperitoneal adoptive transfer of mesenchymal stem cells enhances recovery from acid aspiration acute lung injury in mice
}

Tommaso Mauri ${ }^{1}$, Vanessa Zambelli ${ }^{2}$, Claudia Cappuzzello ${ }^{3}$, Giacomo Bellani ${ }^{2}$, Erica Dander ${ }^{3}$, Marina Sironi $^{4}$, Vittoria Castiglioni ${ }^{5}$, Andrea Doni ${ }^{4}$, Alberto Mantovani ${ }^{4}$, Andrea Biondi ${ }^{2,3}$, Cecilia Garlanda ${ }^{4}$, Giovanna D'amico ${ }^{3}$ and Antonio Pesenti ${ }^{1,6^{*}}$

\footnotetext{
* Correspondence:

antonio.pesenti@unimi.it

${ }^{1}$ Department of Anesthesia, Critical

Care and Emergency, Fondazione

IRCCS Ca' Granda Ospedale

Maggiore Policlinico, Via F. Sforza

35, 20122 Milan, Italy

${ }^{6}$ Department of Pathophysiology and Transplantation, University of

Milan, Milan, Italy

Full list of author information is

available at the end of the article
}

\begin{abstract}
Background: Mesenchymal stem cells (MSCs) might act as fine-tuners of inflammation during acute lung injury. We assessed the effects of adoptive transfer of MSCs in acid aspiration acute lung injury and explored the role of long pentraxin PTX3.

Methods: We conducted a prospective experimental interventional study on wildtype (WT) and PTX3-deficient (PTX3 $3^{-1-}$ ) mice. Acute lung injury was induced in WT and $\mathrm{PTX} 3^{-/-}$mice by instillation of hydrochloric acid into the right bronchus. One hour later, animals received intraperitoneal sterile phosphate-buffered saline (PBS), WT-MSCs $\left(1 \times 10^{6}\right)$ or PTX $3^{-1-}$-MSCs $\left(1 \times 10^{6}\right)$. Twenty-four hours after injury, we measured the effects of treatments on arterial blood gases, wet/dry lung weight (W/ D), CT scan analysis of lung collapse, neutrophils, TNFa and CXCL1 in bronchoalveolar lavage, and plasma PTX3. D-dimer was assayed in 1 week and $\mathrm{OH}$ proline in 2 weeks to track the fibrotic evolution.
\end{abstract}

Results: In 24 h, in comparison to PBS, WT-MSCs improved oxygenation and reduced W/D and alveolar collapse. These effects were associated with decreased concentrations of alveolar neutrophils and cytokines. WT-MSCs increased D-dimer concentration and decreased $\mathrm{OH}$-proline levels, too.

Treatment with PTX3 ${ }^{-1-}$-MSCs ameliorated oxygenation, W/D, and alveolar TNFa, though to a lesser extent than WT-MSCS. PTX $3^{-1-}$-MSCs did not improve lung collapse, neutrophil count, CXCL1, D-dimer, and OH-proline concentrations. The protective effects of WT-MSCs were dampened by lack of endogenous PTX3, too.

Conclusions: In acid aspiration acute lung injury, MSCs improve pulmonary function and limit fibrosis by fine-tuning inflammation. The role of PTX3 in determining MSCs effects might merit further scrutiny.

Keywords: Acute respiratory distress syndrome, Acute lung injury, Stem cells, Pentraxin 3, Acid aspiration syndrome 


\section{Background}

The incidence of the acute respiratory distress syndrome (ARDS) is elevated, and mortality in recent studies still reaches $50 \%$ for the most severe form [1-5]. Moreover, many ARDS survivors develop long-term lung fibrosis, reduced respiratory function, and poor quality of life [1]. At onset, ARDS is characterized by severe hypoxemia and lung edema, caused by dysregulated inflammation $[1,2]$. Overstimulation of leukocytes, cytokine storm, and altered tissue repair are key contributors to ARDS severity, mortality, and long-term morbidity [3]. However, we still lack effective pharmacological therapies that fine-tune these mechanisms [4].

Mesenchymal stem cells (MSCs) are multi-potent cells derived from adult tissues [6]. MSCs secrete multiple molecules, including anti-inflammatory cytokines, growth factors, and anti-microbial peptides, and appear as fine-tuners of host inflammation [6]. Previous studies showed that MSCs administration in animal models of acute lung injury increased the ability of the host to eliminate the agent, regulate neutrophil recruitment, and reverse altered lung permeability, without additional injury [7-10]. In addition, intraperitoneal (i.p.) route for the administration of MSCs was recently described [11]. To our knowledge, the effects of i.p. MSCs have never been assessed in experimental acid aspiration acute lung injury [12]; moreover, the effects of MSCs on the fibrotic long-term evolution of acute lung injury [13] have not been described, and key molecular determinants of MSCs' effects are not fully understood.

In the present study, we tested in a mouse model of acid aspiration acute lung injury the effects of i.p. MSCs on the early acute inflammatory reaction and on the long-term fibrotic evolution [5, 12]. Moreover, we explored the role of pentraxin 3 (PTX3) in mediating MSCs' effects. PTX3 is an acute-phase inflammatory mediator produced by different cell types $[3,14]$ that exerts protective effects in experimental acute lung injury, closely resembling those of MSCs [15]. Previous studies indicated that MSCs produce, store, and secrete PTX3 when activated [16, 17]. The research group of Dr. G. D’Amico generated PTX3-deficient MSCs (PTX3 ${ }^{-/-}$-MSCs) [18], which showed a significant defect in promoting tissue repair in a mice model of wound healing compared to wild-type MSCs (WT-MSCs) [18]. In analogy, we investigated whether PTX3 deficiency in the MSCs and/or at the endogenous level might impact the ability of MSCs to promote short- and longterm recovery from acid aspiration acute lung injury.

The hypothesis of this study was that early treatment with MSCs in a murine model of acid-induced lung injury might exert short- and long-term beneficial effects by modulation of the inflammatory response and that lack of PTX3 in MSCs might reduce their efficacy.

\section{Methods}

\section{Ethics and permissions}

Procedures involving animals and their care were conducted in conformity with the institutional guidelines complying with national and international laws and policies. The experimental protocol was submitted to the Italian Ministry of Health and approved by the Animal Care Unit of the University of Milan-Bicocca, Monza, Italy. 


\section{Isolation of MSCs}

MSCs were isolated from female $\mathrm{C} 57 \mathrm{Bl} / 6 \mathrm{WT}$ mice and from $\mathrm{PTX}^{-1-}$ mice by previously described procedures [18]. Cryopreserved aliquots of MSCs were thawed 5-7 days before the experiments, seeded at 1000-2000 cells $/ \mathrm{cm}^{2}$, and cultured at $37{ }^{\circ} \mathrm{C}$ in a $5 \%$ $\mathrm{CO}_{2}$ atmosphere. On early morning, MSCs were dethatched by trypsin and used fresh for all the experiments performed that same day. Fresh MSCs at passages 5 to 7 were used for the present study. Recent studies showed that PTX3 ${ }^{-1-}$-MSCs were similar to WT-MSCs in their ability to grow spontaneously, undergo mesengenic differentiation, and express common MSCs' markers [18]. As already published, PTX3 ${ }^{-1-}$-MSCs drastically decreased the mitogen-induced proliferation of lymphocyte in a dose-dependent manner similarly to WT-MSCs [19]. Moreover, PTX3 ${ }^{-1-}$-MSCs did not store or release PTX3 while they tended to produce higher levels of tumor necrosis factor-stimulated gene 6 (TSG-6) [19] compared to WT-MSCs (Additional file 1: Figure S1).

\section{Experimental protocol}

Acid aspiration acute lung injury was induced in WT- and PTX3 ${ }^{-1-}$-mice as previously described [12]. Briefly, after intubation, $1.5 \mathrm{ml} / \mathrm{kg}$ of $0.1 \mathrm{M}$ hydrochloric acid was instilled into the right lung, and after $10 \mathrm{~min}$, the animals were extubated and placed in an oxygenated chamber. One hour later (to reproduce possible real life clinical timing), the mice received i.p. injection of sterile phosphate-buffered saline (PBS) or $1 \times 10^{6}$ WT-MSCs or $1 \times 10^{6} \mathrm{PTX3}^{-/-}$-MSCs (all in equal volume of $200 \mu \mathrm{l}$ ).

\section{Experimental design}

Figure 1 shows the experimental design of the study in WT mice. The following measures were performed in all WT mice:

(a) Twenty-four hours after $\mathrm{HCl}$ instillation, the mice were sacrificed and the following analysis were performed (detailed methods are described in Additional files):

- Arterial blood gas analysis for gas exchange

- Wet-to-dry ratio as index of edema

- Micro-CT scan to measure change over time in non-aerated lung tissue expressed as percentage of the whole lung tissue, with more negative values representing larger decrease of alveolar collapse;

- Histopathology examination performed according to previous study [12] evaluating alveolar serofibrinous exudate and alveolar hemorrhage

- Bronchoalveolar lavage for differential cell count, total protein content (with bicinchoninic acid method) and keratinocyte chemoattractant (CXCL1, previously named $\mathrm{KC}$ ), and tumor necrosis factor- $\alpha$ (TNF- $\alpha$ ) were assayed by ELISA

- Blood withdrawal for PTX3 levels measurement in plasma (ELISA assay)

(b)In 1 week from lung injury D-dimer (marker of fibrinolysis) [20] and matrix metalloproteinase 13 (MMP13), an enzyme that participates in collagen degradation [21], were detected by ELISA and by western blot in lungs lysate, respectively.

(c) Two weeks after acid-induced lung injury, the fibrotic evolution was evaluated [22]. In particular, we performed as follows: 


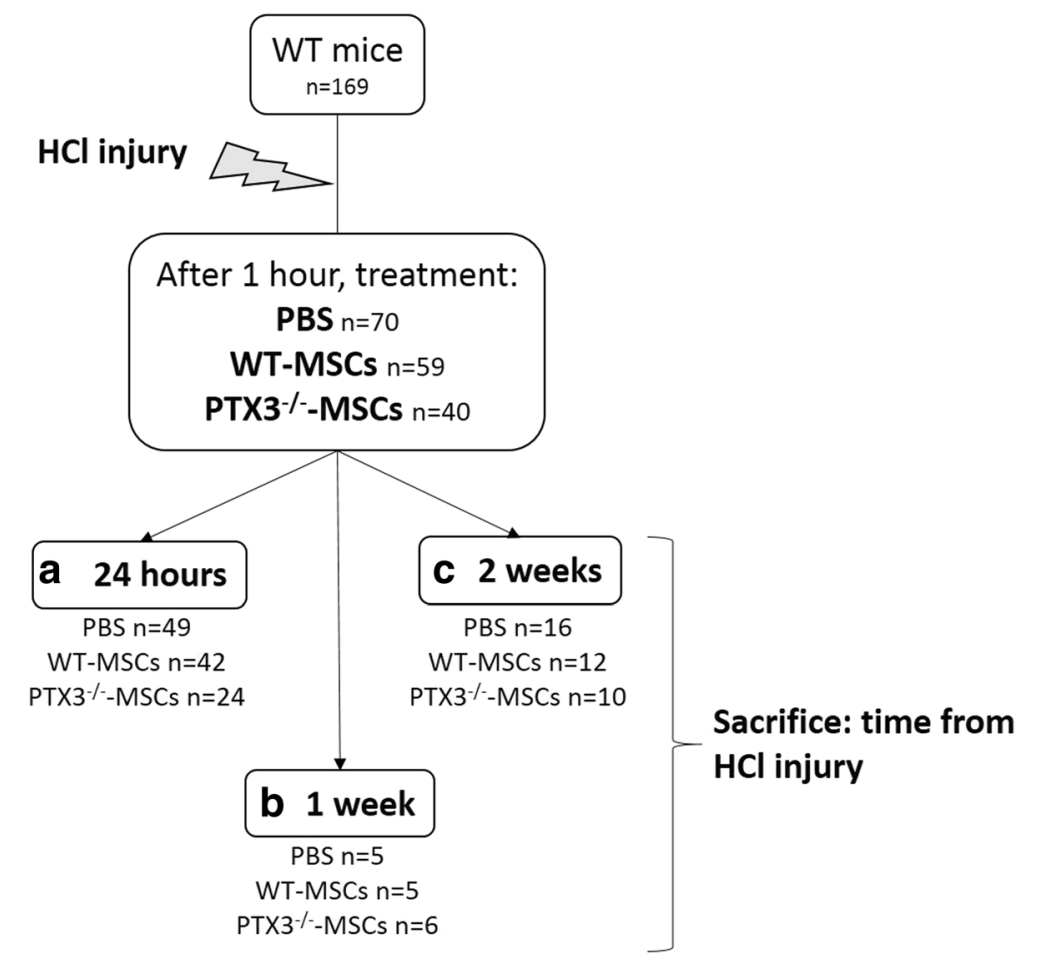

Fig. 1 Experimental design. Experimental groups and number of animals (i.e., WT mice) studied at different time-points (injury, treatment, and sacrifice)

In $\mathrm{PTX}^{-/-}$-mice, instead, we measured only oxygenation and wet-to-dry lung weight ratio in $24 \mathrm{~h}$ and $\mathrm{OH}$-Pro content in 2 weeks. Blinded researchers performed each analysis.

\section{Statistical analysis}

Data are expressed as mean \pm standard deviation if normally distributed and as median (interquartile range) when non-normally distributed. One-way analysis of variance (ANOVA) or Kruskal-Wallis and Dunnett's or Dunn's post hoc tests vs. PBS group were used to assess differences between treatment effects in WT mice, as appropriate. Differences in physiologic variables measured in the right vs. left lung were assessed by $t$ test or Mann-Whitney $U$ test, as appropriate. $p<0.05$ was considered statistically significant.

Detailed methods can be found in the Additional file 1 of this article.

\section{Results}

Mesenchymal stem cells enhance short- and long-term recovery from experimental acid aspiration acute lung injury

In $24 \mathrm{~h}$, i.p. administration of WT-MSCs $1 \mathrm{~h}$ after induction of acid aspiration acute lung injury significantly improved arterial oxygenation and decreased the alveolar-arterial oxygen gradient in WT-mice in comparison to PBS $(p<0.05$ and $p=0.001$, respectively) (Fig. 2a and b), without modification of $\mathrm{PaCO}_{2}$ and even in presence of slightly worse $\mathrm{pH}$ values (Additional file 1: Table S1). Early improvement in oxygenation 

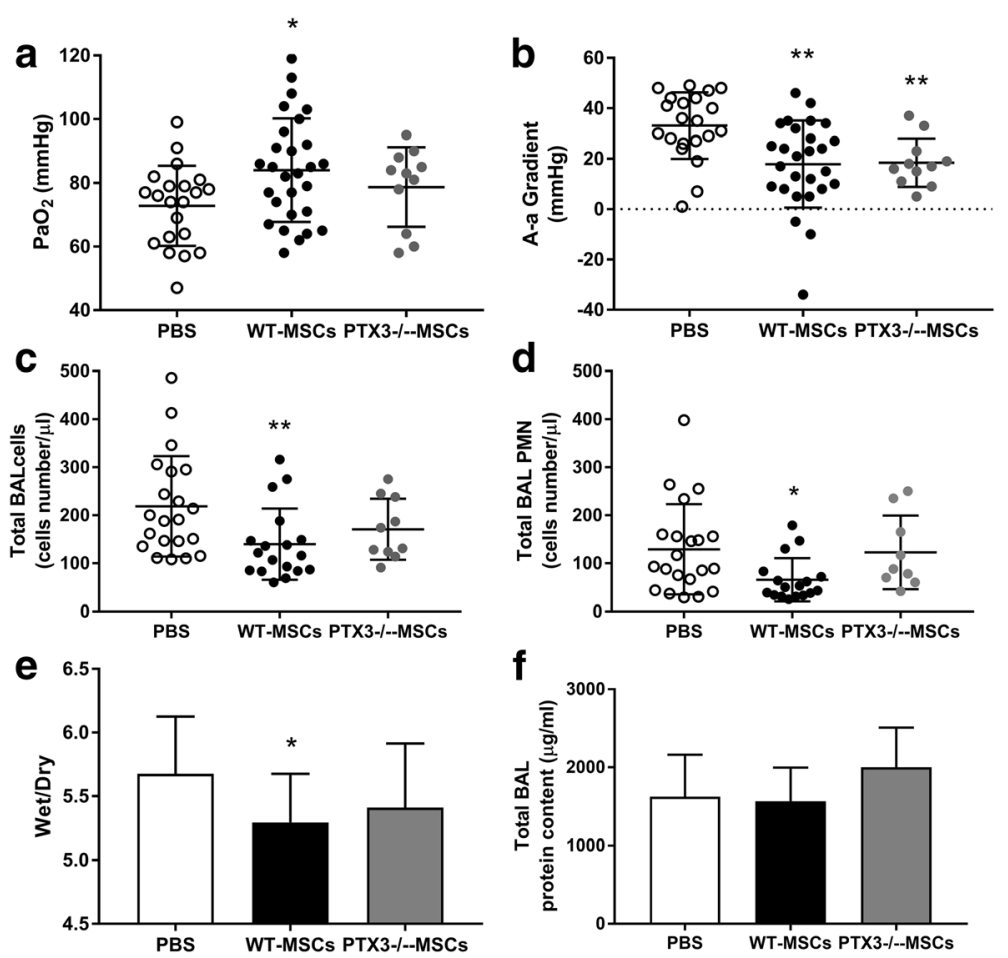

Fig. 2 Early effects of mesenchymal stem cells (MSCs) on oxygenation, lung edema, and alveolar inflammatory cells in acid aspiration acute lung injury. Wild-type MSCs ameliorated arterial oxygen tension (a) and alveolar-arterial oxygen gradient (b) in experimental model of acid aspiration lung injury in $24 \mathrm{~h}$, as well as PTX3-deficient MSCs, albeit to a lesser extent (Kruskal-Wallis $p<0.05[\mathrm{~A}]$ and $p=0.001$ [B]; Dunn's post hoc ${ }^{*} p<0.05$ and ${ }^{* *} p<0.01$ vs. PBS. PBS $n=21$; WT-MSCs $n=28$; PTX3 ${ }^{-1-}-$ MSCs $n=11$ ). Total cell count (c) and total neutrophil (PMN) count (d) in the broncho-alveolar lavage (BAL) were decreased by WT-MSCs but not by PTX3 ${ }^{-1-}$-MSCs in experimental groups in $24 \mathrm{~h}$ (Kruskal-Wallis $p<0.05$ [C] and $p=0.01$ [D]; Dunn's post hoc ${ }^{*} p<0.05$ and ${ }^{* *} p<0.01$ vs. PBS. PBS $n=21$; WT-MSCs $n=17$; PTX $\left.3^{-1-}{ }^{-M S C s} n=9\right)$. WT-MSCs significantly reduced lung edema (c), as measured by wet-to-dry lung weight ratio (wet/dry) in the experimental groups in $24 \mathrm{~h}$ (ANOVA $p<0.05$ [e]; *Dunnett's post hoc $p<0.05$ vs. PBS. PBS $n=21$; WT-MSCs $n=18$; PTX3 ${ }^{-1-}$-MSCs $n=10$ ). No difference was seen in BAL total protein concentrations (f) (PBS $n=22 ;$ WT-MSCs $n=$ 20; $\mathrm{PTX}^{-1-}$-MSCS $n=10$ ). (PBS = acid aspiration acute lung injury + intraperitoneal (i.p.) PBS treatment in $1 \mathrm{~h}$; WT-MSCs = acid aspiration acute lung injury + i.p. wild-type MSCs treatment in $1 \mathrm{~h}$; PTX3 ${ }^{-1-}$-MSCs = acid aspiration acute lung injury + i.p. PTX3-deficient MSCs treatment in $1 \mathrm{~h}$ )

yielded by WT-MSCs was likely obtained by reduction of lung edema: in fact, the lungs' wetto-dry ratio in $24 \mathrm{~h}$ was decreased by WT-MSCs in comparison to PBS $(P<0.05)$ (Fig. 2e). Similarly, micro-CT scan analysis showed that the extent of lung collapse significantly decreased between 1 and $24 \mathrm{~h}$ in WT mice treated by WT-MSCs $(p=0.01)$, likely indicating decreased superimposed weight from reduced lung edema (Table 1 and Fig. 3), but not in those treated by PBS. Histology performed in $24 \mathrm{~h}$ showed decreased disruption of lung structures in mice treated by WT-MSCs in comparison to PBS (Table 1), even though this difference did not reach statistical significance. BAL total protein concentrations were left unchanged by WT-MSCs treatment (Fig. 2f). Mice treated by WT-MSCs, indeed, showed significant reduction of total cell count in BAL fluid in $24 \mathrm{~h}$ and substantial dampening of neutrophil recruitment into the alveoli ( $p<0.05$ for both; Fig. 2c, d) in comparison to PBS. Accordingly, levels of proinflammatory cytokines (i.e., CXCL1 and TNF- $\alpha$ ) in BAL fluid were significantly reduced by WT-MSCs $(p<0.05$ and $p<0.01$, respectively), but not by PBS (Table 1). Interestingly, circulating PTX3 was reduced in WT-mice treated by WT-MSCs (albeit non-significantly) and not in WT-mice treated by PTX3-deficient MSCs (Table 1). 


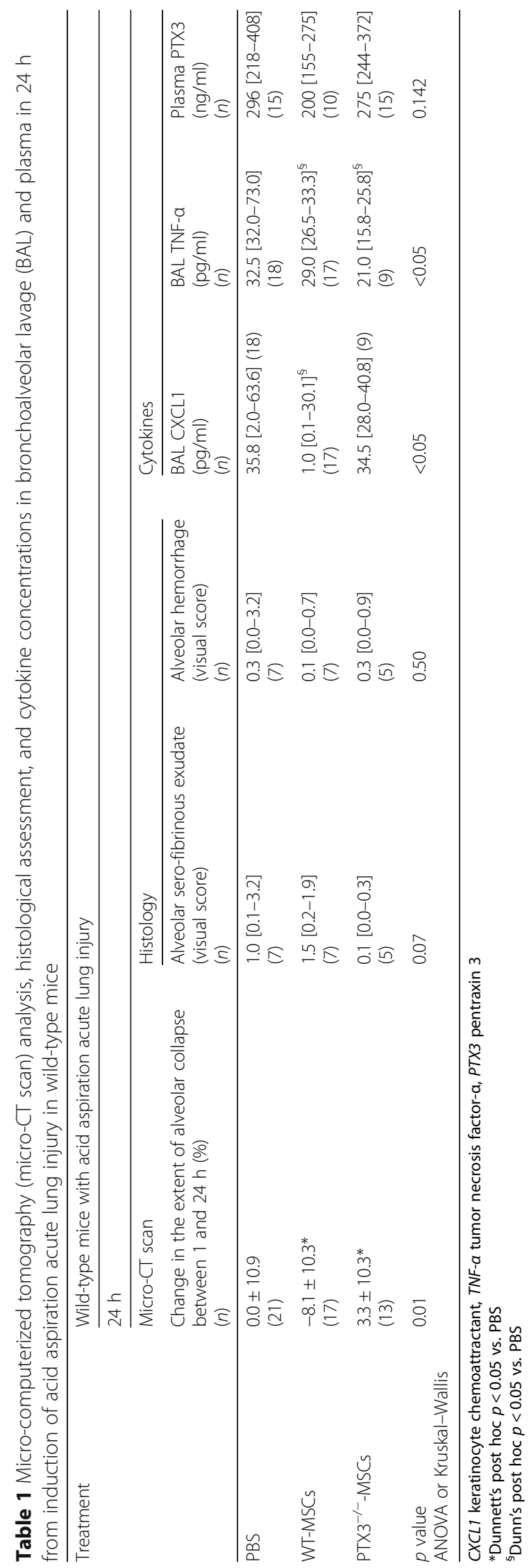




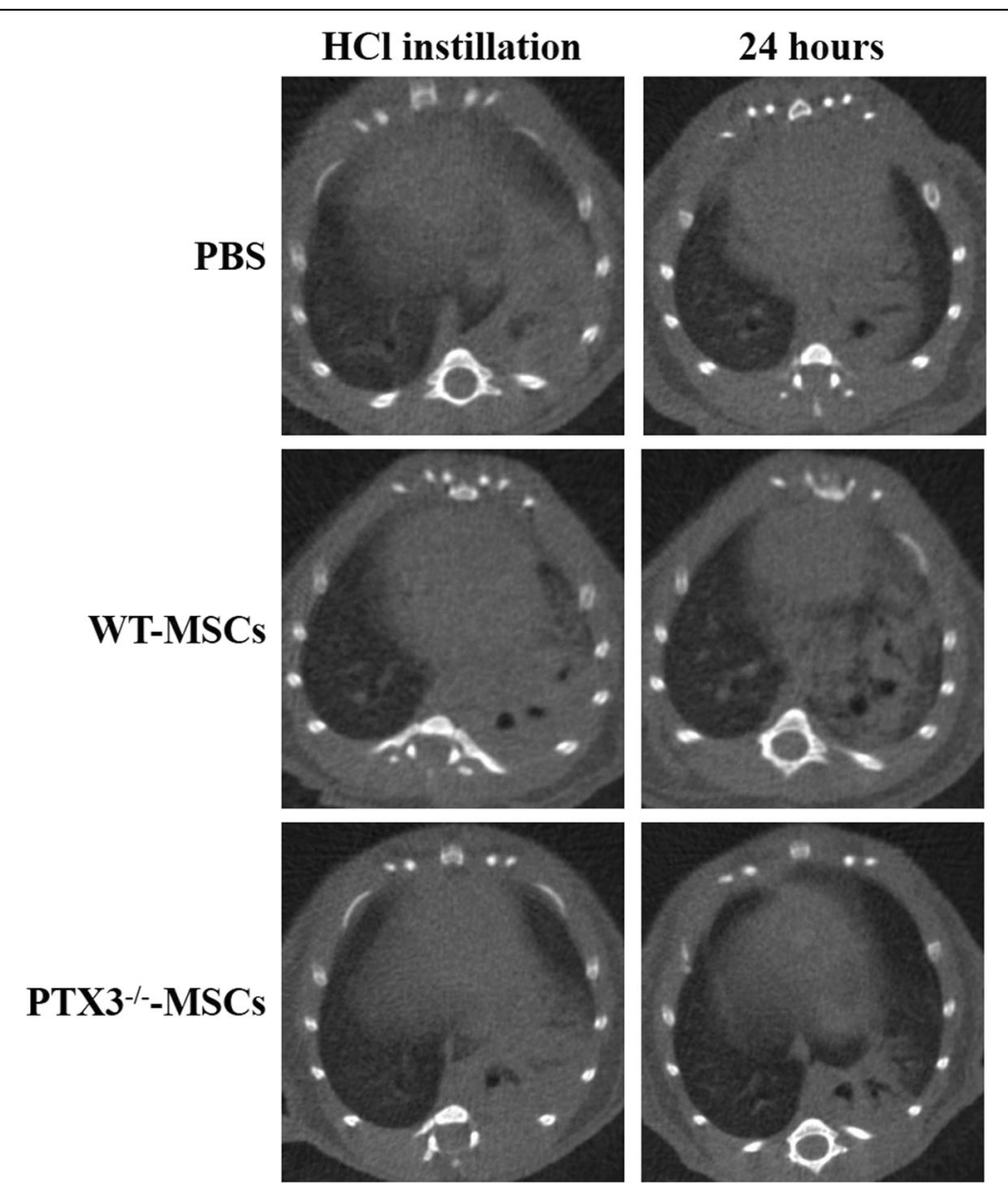

Fig. 3 Short-term effects of MSCs: CT scan images. Chest $C T$ images from representative study animals treated by PBS, WT-MSCs, or PTX3 ${ }^{-1-}$-MSCs in $1 \mathrm{~h}$ after $\mathrm{HCl}$ instillation and $24 \mathrm{~h}$ showing reduced radiologic signs of lung edema and collapse, especially in the right lung

In this study, we showed that treatment by i.p. WT-MSCs administered $1 \mathrm{~h}$ after acid aspiration attenuated the evolution of fibrosis, as demonstrated by lower collagen deposition (OH-Pro assay) in 2 weeks (Fig. 4a) in comparison to mice treated by PBS. In 1 week, D-dimer concentration was significantly increased in the lungs of mice treated with WT-MSCs $(p<0.001$, Fig. $4 \mathrm{~b})$ in comparison to PBS, suggesting that dampening of long-term fibrotic evolution might have followed both reduced inflammation and enhanced fibrinolysis by WT-MSCs in the days after injury.

\section{Systemic deployment of WT-MSCs in $24 \mathrm{~h}$}

In an effort to evaluate whether i.p. WT-MSCs migrate systemically in mice with acid aspiration acute lung injury, we performed western blot analysis to detect $\mathrm{GFP}^{+}$WT-MSCs presence in the lungs, spleen, liver, and peritoneal lavage in $24 \mathrm{~h}$. Additional file 1: Figure S3 shows actual blots with no apparent signal of $\mathrm{GFP}^{+}$WT-MSCs presence in the lungs, spleen, and liver as opposed to positive controls. In the peritoneal lavage, instead, WTMSCs were still present in $24 \mathrm{~h}$ but by lower intensity, probably because, as previously shown [23], they formed aggregates and adhered to the peritoneal cavity walls. 

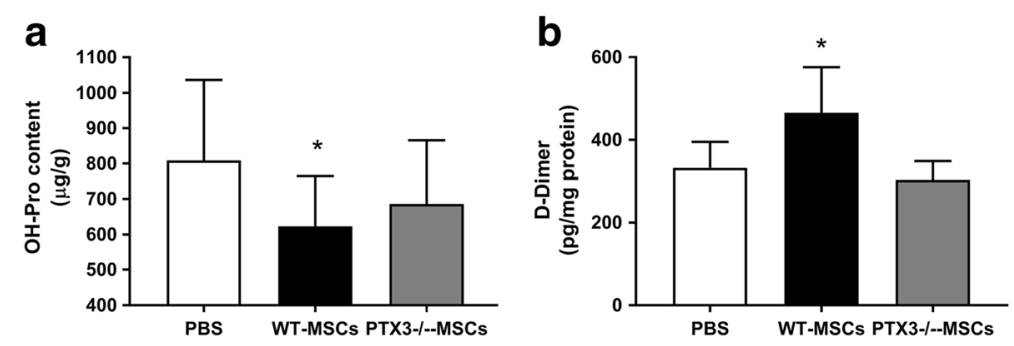

Fig. 4 Effects of WT-MSCs on the fibrotic evolution of acid aspiration acute lung injury. Collagen deposition (OH-proline assay) (a) in lung tissue in the experimental groups in 2 weeks: WT-MSCs decreased presence of fibrotic tissue while PTX3 ${ }^{-1-}$-MSCs could not (ANOVA $p<0.05$ [A]; *Dunnett's post hoc $p<0.05$ vs. PBS. PBS $n=16$; WT-MSCs $n=12$; PTX3 ${ }^{-1-}$-MSCs $\left.n=10\right)$ ). Decreased fibrotic evolution by WT-MSCs was likely mediated by improved fibrinolysis (b) in the lungs over the days following acid aspiration acute lung injury (ANOVA $p<0.01$ [B]; *Dunnett's post hoc $p<0.05$ vs. PBS. PBS $n=5$; WT-MSCs $n=5$; PTX3 ${ }^{-1-}{ }^{-M S C s} n=6$ ) (PBS = acid aspiration acute lung injury + intraperitoneal (i.p.) PBS treatment in $1 \mathrm{~h}$; WT-MSCs = acid aspiration acute lung injury + i.p. wild-type MSCs treatment in $1 \mathrm{~h}$; $\mathrm{PTX}^{-1-}-\mathrm{MSCs}=$ acid aspiration acute lung injury + i.p. PTX3-deficient MSCs treatment in $1 \mathrm{~h}$ )

Lack of PTX3 in MSCs reduces early and long-term protection from acid aspiration acute lung injury

$\mathrm{PTX}^{-1-}$-MSCs were isolated from the bone marrow of PTX3-knockout mice. The extensive characterization of $\mathrm{PTX}^{-/-}$-MSCs demonstrated that these cells did not seem to modify their in vitro phenotypical and functional properties [18] (Additional file 1: Figure S1). In $24 \mathrm{~h}$, treatment by $\mathrm{PTX}^{-/-}-\mathrm{MSCs}$ ameliorated oxygenation only to a lesser extent than WT-MSCs (Fig. 2). Reduced short-term effects on oxygenation in comparison to WT-MSCs were paralleled by less effective reduction of wet-to-dry lung weight ratio by PTX3 ${ }^{-1-}$-MSCs (Fig. 2a, b, e) and the absence of effects of PTX3deficient cells on radiological signs of regional lung collapse and edema (Table 1). Histology found reduction of lung injury, albeit non-significant (Table 1). In summary, $\mathrm{PTX}^{-1-}$-MSCs seemed less effective than WT-MSCs in limiting formation of lung edema in $24 \mathrm{~h}$ after acid aspiration. At variance from WT-MSCs, treatment with PTX3 ${ }^{-1-}$-MSCs did not reduce total cell and neutrophil count (Fig. 2) as well as CXCL1 levels in the alveolar space (Table 1). Thus, the more limited effectiveness of PTX3 ${ }^{-1}$-MSCs in enhancing lung recovery after acid aspiration acute lung injury might have been related to ineffective reduction of the acute inflammatory processes. Moreover, $\mathrm{PTX}^{-1-}$-MSCs could not modulate fibrinolysis in the days following injury nor impact the long-term fibrotic evolution, as demonstrated by unchanged levels of D-dimer and $\mathrm{OH}$-proline in comparison to PBS (Fig. 4a, b). However, WT- and PTX3 ${ }^{-1-}$-MSCs did not seem to modulate activity of MMP13 in 1 week (Additional file 1: Figure S2) to impact remodeling and fibrosis.

Effects of study treatments on PTX3 knockout mice with acid aspiration acute lung injury Extent of lung injury was similar between WT and PTX3 ${ }^{-1-}$ mice $(t$ test in $24 \mathrm{~h}$ in WTmice + PBS vs. PTX3 ${ }^{-1-}$-mice + PBS: $\mathrm{PaO}_{2}, p=0.151$; wet-to-dry lung weight, $p=$ 0.099). When administered to PTX $3^{-1-}$-mice: WT-MSCs improved lung function and reduced fibrosis, but the difference with PBS was non-significant (Additional file 1: Table S3); $\mathrm{PTX}^{-1-}$-MSCs induced a further non-significant reduction of the alveolararterial gradient and of wet-to-dry lung weight ratio, while $\mathrm{PaO}_{2}$ and fibrosis worsened 
in comparison to WT-MSCs (Additional file 1: Table S3). Thus, endogenous PTX3 might collaborate in the protective effects of WT-MSCs from fibrosis, while it might limit their effectiveness in reducing lung edema.

More results are provided in the Additional files of this article.

\section{Discussion}

Study's main findings can be summarized as follows: WT-MSCs dampen short- and long-term sequelae of acid aspiration acute lung injury in mice in terms of improved oxygenation, reduced edema causing lung collapse, and reduced fibrotic evolution, likely by fine-tuning the acute inflammatory reaction and the subsequent fibrinolysis and tissue repair process; moreover, lack of PTX3 gene in MSCs and in the injured host might reduce the beneficial effects of MSCs.

In the present study, we administered i.p. WT-MSCs $1 \mathrm{~h}$ after intratracheal instillation of hydrochloric acid, potentially reproducing real-life treatment of ARDS caused by aspiration of gastric contents [1, 12], one of the major direct causes of ARDS [24, 25] with a mortality rate around $35-40 \%$ and significant long-term fibrosis [1]. In $24 \mathrm{~h}$ from injury, we could show multiple short-term beneficial effects of WT-MSCs: as previously shown $[7,26]$, MSCs seemed to reduce the early inflammatory reaction in the lungs and to avoid excessive response and additional damage. In our study, indeed, MSCs dampened leukocyte trafficking through the alveolar-epithelial barrier as well as their activation and release of primary inflammatory cytokines. In turn, as testified by oxygenation, wet-to-dry and CT scan data, this led to decreased accumulation and/or improved clearance of lung edema and inflammatory cells in the alveolar and thirdspace compartments and to attenuated extent of alveolar collapse. However, histology did not improve after WT-MSCs administration, maybe due to insufficient numerosity; similarly, protein content in BAL was not reduced by MSCs, but this could have followed direct extravasation after acid-induced physical disruption of the alveolar-epithelial integrity. In our model, both lungs showed physiologic alterations, thus indicating that the left lung could completely compensate for the ventilation needs of the animals (Additional file 1: Table S2) [27].

In 2 weeks from acute lung injury onset, we also showed decreased long-term collagen deposition in the lungs associated with treatment by WT-MSCs. Moreover, the long-term reduction of fibrosis was preceded by increased fibrinolysis in 1 week. Our data, in keeping with recent literature $[7,9,20]$, seem to suggest that the beneficial effects of MSCs on the fibrotic evolution of acute lung injury might include reduction of the acute-phase inflammatory reaction and reduced fibrosis in 2 weeks. Moreover, decreased respiratory effort during the early phases induced by improved gas exchange could have reduced interstitial lung edema [28] and the risk of additional ventilationinduced lung injury (VILI) and fibrosis [13]. In summary, it would be tempting to say that ours and the previous data indicate that MSCs might be regarded as personalized modular therapies limiting short- and long-term acute lung injury severity by finetuning inflammation and tissue remodeling. However, to date, whether these hypotheses hold true and will translate in improved mortality and long-term quality of life in human ARDS remains to be determined.

PTX3 is a marker of severity in human ARDS [29], and experimental models showed that PTX3 is as key determinant of the evolution, morbidity, and mortality of acute 
lung injury [30, 31]. A recent study by Cappuzzello and colleagues showed that while WT-MSCs improved tissue repair in experimental wound healing, $\mathrm{PTX}^{-/-}$-MSCs could not [18]. Similarly, we showed that the early dampening of leukocyte migration and release of pro-inflammatory cytokines in the injured lungs by WT-MSCs could not be replicated when $\mathrm{PTX}^{-/-}$-MSCs were adopted. This likely led to poorer effects on oxygenation and wet/dry ratios and no improvement in the CT scan analysis of lung collapse as well as no decrease in inflammatory cells and acute-phase primary cytokines in the BAL. The positive effect of PTX3 ${ }^{-/-}$-MSCs treatment on the TNF- $\alpha$ levels may depend on the anti-inflammatory role of TSG6 [32, 33], which is highly expressed in $\mathrm{PTX}^{-1-}$-MSC (Additional file 1: Figure S1). Previous studies in a mice model of bilateral acid aspiration lung injury showed that interaction between PTX3 and Pselectin is crucial for regulation of leukocyte recruitment with consequences on cytokine production and lung injury [23], and similar mechanisms might underlie lack of lung protection by $\mathrm{PTX3}^{-/-}$-MSCs. Moreover, we described that long-term fibrinolysis and subsequent fibrotic evolution could not be prevented by $\mathrm{PTX}^{-/-}$-MSCs, possibly suggesting PTX3-mediated enhancement of lung tissue repair by WT-MSCs $[18,20]$. On the other hand, our data indicate that the beneficial effects exerted by WT-MSCs are associated with a reduction in plasma PTX3, as if improvement of lung injury preceded modification of endogenous PTX3 production. However, lack of endogenous PTX3 seemed to reduce WT-MSCs effects (Additional file 1: Table S3), maybe by impairment of local cell-to-cell interaction. Our results do not generate a clear hypothesis on the role of PTX3 as molecular determinant of the lung protection exerted by MSCs, and further studies are warranted, maybe exploring other etiologies and time-points.

In our study, we could not detect presence of WT-MSCs in the liver, spleen, or lungs in $24 \mathrm{~h}$, while in keeping with previous findings [34], a signal was still present in peritoneal lavage (Additional file 1: Figure S3). On the other hand, since levels of circulating PTX3 were lower and lung fibrinolysis was increased after administration of WTMSCs, we might speculate possible migration and direct effect of MSCs at the site of injury but this cannot be concluded with any confidence. In summary, our data are not definitive to elucidate whether i.p. WT-MSCs act through paracrine vs. direct mechanisms.

This study suffers by a number of relevant limitations: as most of the measures required sacrifice of the animals (e.g., BAL), we could not assess in the same animal all the effects at different time-points but each effect was assessed in a subset of animals receiving the same injury and therapy, which might have introduced some heterogeneity. We only examined three time-points (i.e., $24 \mathrm{~h}$ and 1 and 2 weeks), which might have prevented us from description of other effects of WT-MSCs or PTX ${ }^{-1-}$-MSCs in acid aspiration acute lung injury. Apart from resources limitation, our choice was based on previous observations on the time-course of the studied animal model [12]. We described reduced effectiveness of WT-MSCs induced by lack of PTX3 only in a murine model of non-infective acid aspiration lung injury and translation of these findings to other etiologies (e.g., infective pulmonary ARDS) and/or to the clinical setting warrants extreme caution. While we could determine significant effects of PTX3 presence in MSCs in 1 week to modulate fibrosis, the downstream effects of PTX3 presence in MSCs during the early acute phase (e.g., modulation of leukocyte recruitment by 
binding with P-selectin) remains to be elucidated. We did not evaluate the alteration of the alveolar-capillary permeability from a more molecular point of view (such as expression of tight or adherent junction proteins), but only by measuring the lung edema following such alterations (wet-to-dry ratio and the CT scan analysis). Besides the standard histological analysis, we did not perform more quantitative approach using stereological assessment of the tissue injury, and this might have limited our possibilities to describe more significant differences. This is a preliminary study: the small number of mice used and lack of other administration routes could have reduced significant differences. The experiments on $\mathrm{PTX}^{-/-}$mice were subsequent and separate from those on WT mice. Finally, volume of fluid instilled i.p. (i.e., $200 \mu \mathrm{l}$ ) might have induced cardiovascular impairment favoring pulmonary edema.

\title{
Conclusions
}

The results presented here suggest that i.p. adoptive transfer of MSCs enhances shortand long-term lung recovery when cells are administered $1 \mathrm{~h}$ after onset of acute lung injury. PTX3, an acute-phase inflammatory mediator, might play a role in the lung protection exerted by MSCs, in particular against fibrosis, but this needs further clarification. Studies on the molecular mechanisms of actions of MSCs as well as on the risks associated with their administration should still proceed in parallel with ongoing translational studies [35]. In particular, PTX3 genetic polymorphisms have been associated with risk of microbial infections [36-38], and it will be important to assess whether PTX3 polymorphisms are associated with outcome in ARDS and in clinical trials aimed to assess the potential of MSCs.

\section{Additional file}

Additional file 1: Detailed methods and additional results. (DOCX $838 \mathrm{~kb}$ )

\begin{abstract}
Abbreviations
ANOVA: One-way analysis of variance; ARDS: Acute respiratory distress syndrome; BAL: Bronchoalveolar lavage; CXCL1: Keratinocyte chemoattractant; GFP+: Green fluorescent protein-positive; i.p.: Intraperitoneal; MMP13: Matrix metalloproteinase 13; MSCs: Mesenchymal stem cells; OH-Pro: Hydroxyproline; PBS: Phosphate-buffered saline; PTX3: Pentraxin 3; PTX3 ${ }^{-1-}$-MSCs: PTX3-deficient MSCs; TNF-a: Tumor necrosis factor-a; TSG-6: Tumor necrosis factorstimulated gene 6; WT-MSCs: Wild-type MSCs
\end{abstract}

\section{Acknowledgements}

We are thankful to all the personnel working in the University of Milan-Bicocca, San Gerardo Hospital and Humanitas Institute for the continuous support and collaboration.

\section{Funding}

The present study was supported by departmental fundings (AP); by the Ministry of University and research (MIUR, Rome, Italy) (TM, VZ, AP); by Associazione Italiana Ricerca sul Cancro and Italian Ministry of Health (CC, ED, GD); by the Fondazione Cariplo and Italian Ministry of Health (Ricerca Finalizzata) (AM); and by the Italian Ministry of Health (Ricerca Finalizzata) (CG).

Availability of data and materials

The datasets supporting the conclusions of this article are included within the article (and its additional file).

TM, VZ, CG, GD, and AP made substantial contributions to the conception and design of the work. All authors performed the data acquisition, analysis, and interpretation for the work. TM, VZ, CC, AM, AB, CG, GD, and AP drafted the work and revised it critically for important intellectual content. The final approval of the version submitted for publication was carried out by all authors. TM, VZ, and AP established the accountability for all aspects of the work in ensuring that questions related to the accuracy or integrity of any part of the work are appropriately investigated and resolved. All authors read and approved the final manuscript. 
Authors' information

Authors' qualifications: TM: MD; VZ: PhD; CC: PhD; GB: MD PhD; ED: PhD; MS: PhD; VC: PhD; AD: PhD; AM: MD; AB: MD; CG: PhD; GD: PhD; AP: MD

\section{Competing interests}

AM is an inventor of patents on PTX3 and receives royalties related to PTX3 reagents. The other authors do not have any conflict of interest to disclose.

\section{Author details}

'Department of Anesthesia, Critical Care and Emergency, Fondazione IRCCS Ca' Granda Ospedale Maggiore Policlinico, Via F. Sforza 35, 20122 Milan, Italy. ${ }^{2}$ School of Medicine and Surgery, University of Milan-Bicocca, Monza, Italy. ${ }^{3}$ Research Center 'M. Tettamanti', Fondazione MBBM/San Gerardo Hospital, Monza, Italy. ${ }^{4}$ Humanitas Clinical and Research Center, Rozzano, MI, Italy. ${ }^{5}$ Mouse and Animal Pathology Lab, Fondazione Filarete, Milan, Italy. ${ }^{6}$ Department of Pathophysiology and Transplantation, University of Milan, Milan, Italy.

Received: 2 September 2016 Accepted: 21 February 2017

Published online: 06 March 2017

\section{References}

1. Matthay MA, Ware LB, Zimmerman GA (2012) The acute respiratory distress syndrome. J Clin Invest 122(8):2731-2740

2. Jabaudon M, Blondonnet R, Roszyk L, Bouvier D, Audard J, Clairefond G, Fournier M, Marceau G, Déchelotte P, Pereira B, Sapin V, Constantin JM (2015) Soluble receptor for advanced glycation end-products predicts impaired alveolar fluid clearance in acute respiratory distress syndrome. Am J Respir Crit Care Med 192(2):191-199

3. Calfee CS, Delucchi K, Parsons PE, Thompson BT, Ware LB, Matthay MA, the NHLBI ARDS Network (2014) Subphenotypes in acute respiratory distress syndrome: latent class analysis of data from two randomised controlled trials. Lancet Respir Med 2(8):611-620

4. Gotts JE, Matthay MA (2014) Treating ARDS: new hope for a tough problem. Lancet Respir Med 2(2):84-85

5. Zambelli V, Bellani G, Borsa R, Pozzi F, Grassi A, Scanziani M, Castiglioni V, Masson S, Decio A, Laffey JG, Latini R, Pesenti A (2015) Angiotensin-(1-7) improves oxygenation, while reducing cellular infiltrate and fibrosis in experimental acute respiratory distress syndrome. Intensive Care Med Exp 3(1):44

6. Zhu YG, Feng XM, Abbott J, Fang XH, Hao Q, Monsel A, Qu JM, Matthay MA, Lee JW (2014) Human mesenchymal stem cell microvesicles for treatment of Escherichia coli endotoxin-induced acute lung injury in mice. Stem Cells 32(1):116-125

7. Horie S, Masterson C, Devaney J, Laffey JG (2016) Stem cell therapy for acute respiratory distress syndrome: a promising future? Curr Opin Crit Care 22(1):14-20

8. Lee JW, Fang X, Gupta N, Serikov V, Matthay MA (2009) Allogenic human mesenchymal stem cells for treatment of E. coli endotoxin-induced acute lung injury in the ex vivo perfused human lung. Proc Natl Acad Sci U S A 106:16357-16362

9. Curley GF, Ansari B, Hayes M, Devaney J, Masterson C, Ryan A, Barry F, O'Brien T, Toole DO, Laffey JG (2013) Effects of intratracheal mesenchymal stromal cell therapy during recovery and resolution after ventilator-induced lung injury. Anesthesiology 118(4):924-932

10. Asmussen S, Ito H, Traber DL, Lee JW, Cox RA, Hawkins HK, McAuley DF, McKenna DH, Traber LD, Zhuo H, Wilson J, Herndon DN, Prough DS, Liu KD, Matthay MA, Enkhbaatar P (2014) Human mesenchymal stem cells reduce the severity of acute lung injury in a sheep model of bacterial pneumonia. Thorax 69(9):819-25

11. Zheng G, Huang L, Tong H, Shu Q, Hu Y, Ge M, Deng K, Zhang L, Zou B, Cheng B, Xu J (2014) Treatment of acute respiratory distress syndrome with allogeneic adipose-derived mesenchymal stem cells: a randomized, placebocontrolled pilot study. Respir Res 15:39

12. Amigoni M, Bellani G, Scanziani M, Masson S, Bertoli E, Radaelli E, Patroniti N, Di Lelio A, Pesenti A, Latini R (2008) Lung injury and recovery in a murine model of unilateral acid aspiration: functional, biochemical, and morphologic characterization. Anesthesiology 108(6):1037-1046

13. Cabrera-Benitez NE, Laffey JG, Parotto M, Spieth PM, Villar J, Zhang H, Slutsky AS (2014) Mechanical ventilationassociated lung fibrosis in acute respiratory distress syndrome: a significant contributor to poor outcome. Anesthesiology 121(1):189-198

14. Mauri T, Coppadoro A, Bombino M, Bellani G, Zambelli V, Fornari C, Berra L, Bittner EA, Schmidt U, Sironi M, Bottazzi B, Brambilla P, Mantovani A, Pesenti A (2014) Alveolar pentraxin 3 as an early marker of microbiologically confirmed pneumonia: a threshold-finding prospective observational study. Crit Care 18(5):562

15. Daigo K, Mantovani A, Bottazzi B (2014) The yin-yang of long pentraxin PTX3 in inflammation and immunity. Immunol Lett 161(1):38-43

16. Chiellini C, Cochet O, Negroni L, Samson M, Poggi M, Ailhaud G, Alessi MC, Dani C, Amri EZ (2008) Characterization of human mesenchymal stem cell secretome at early steps of adipocyte and osteoblast differentiation. BMC Mol Biol 9:26

17. Lee MJ, Kim J, Kim MY, Bae YS, Ryu SH, Lee TG, Kim JH (2010) Proteomic analysis of tumor necrosis factor-alphainduced secretome of human adipose tissue-derived mesenchymal stem cells. J Proteome Res 9(4):1754-1762

18. Cappuzzello C, Doni A, Dander E, Pasqualini F, Nebuloni M, Bottazzi B, Mantovani A, Biondi A, Garlanda C, D'Amico G (2016) Mesenchymal stromal cell-derived PTX3 promotes wound healing via fibrin remodeling. J Invest Dermatol 136(1):293-300

19. Walter J, Ware LB, Matthay MA (2014) Mesenchymal stem cells: mechanisms of potential therapeutic benefit in ARDS and sepsis. Lancet Respir Med 2(12):1016-1026

20. Doni A, Musso T, Morone D, Bastone A, Zambelli V, Sironi M, Castagnoli C, Cambieri I, Stravalaci M, Pasqualini F, Laface I, Valentino S, Tartari S, Ponzetta A, Maina V, Barbieri SS, Tremoli E, Catapano AL, Norata GD, Bottazzi B, Garlanda C, Mantovani A (2015) An acidic microenvironment sets the humoral pattern recognition molecule PTX3 in a tissue repair mode. J Exp Med 212(6):905-925 
21. Juncker-Jensen A, Lund LR (2011) Phenotypic overlap between MMP-13 and the plasminogen activation system during wound healing in mice. PLoS One 6(2):e16954

22. Tager AM, Kradin RL, LaCamera P, Bercury SD, Campanella GSV, Leary CP, Polosukhin V, Zhao LH, Sakamoto H, Blackwell TS, Luster AD (2004) Inhibition of pulmonary fibrosis by the chemokine IP-10/CXCL10. Am J Respir Cell Mol Biol 31:395-404

23. Bazhanov N, Ylostalo JH, Bartosh TJ, Tiblow A, Mohammadipoor A, Foskett A, Prockop DJ (2016) Intraperitoneally infused human mesenchymal stem cells form aggregates with mouse immune cells and attach to peritoneal organs. Stem Cell Res Ther 10:7-27

24. Raghavendran K, Nemzek J, Napolitano LM, Knight PR (2011) Aspiration-induced lung injury. Crit Care Med 39(4):818-826

25. Bellani G, Laffey JG, Pham T, Fan E, Brochard L, Esteban A, Gattinoni L, van Haren F, Larsson A, McAuley DF, Ranier M, Rubenfeld G, Thompson BT, Wrigge H, Slutsky AS, Pesenti A, LUNG SAFE Investigators; ESICM Trials Group (2016) Epidemiology, patterns of care, and mortality for patients with acute respiratory distress syndrome in intensive care units in 50 countries. JAMA 315(8):788-800

26. Monsel A, Zhu YG, Gennai S, Hao Q, Hu S, Rouby JJ, Rosenzwajg M, Matthay MA, Lee JW (2015) Therapeutic effects of human mesenchymal stem cell-derived microvesicles in severe pneumonia in mice. Am J Respir Crit Care Med 192(3):324-36

27. Amigoni M, Bellani G, Zambelli V, Scanziani M, Farina F, Fagnani L, Latini R, Fumagalli R, Pesenti A (2013) Unilateral acid aspiration augments the effects of ventilator lung injury in the contralateral lung. Anesthesiology 119(3):642-651

28. Yoshida T, Roldan R, Beraldo MA, Torsani V, Gomes S, De Santis RR, Costa EL, Tucci MR, Lima RG, Kavanagh BP, Amato MB (2016) Spontaneous effort during mechanical ventilation: maximal injury with less positive endexpiratory pressure. Crit Care Med 44(8):e678-88

29. Mauri T, Masson S, Pradella A, Bellani G, Coppadoro A, Bombino M, Valentino S, Patroniti N, Mantovani A, Pesenti A, Latini R (2010) Elevated plasma and alveolar levels of soluble receptor for advanced glycation endproducts are associated with severity of lung dysfunction in ARDS patients. Tohoku J Exp Med 222(2):105-112

30. Han B, Ma X, Zhang J, Zhang Y, Bai X, Hwang DM, Keshavjee S, Levy GA, McGilvray I, Liu M (2012) Protective effects of long pentraxin PTX3 on lung injury in a severe acute respiratory syndrome model in mice. Lab Invest 92(9):1285-1296

31. Han B, Haitsma JJ, Zhang Y, Bai X, Rubacha M, Keshavjee S, Zhang H, Liu M (2011) Long pentraxin PTX3 deficiency worsens LPS-induced acute lung injury. Intensive Care Med 37(2):334-42

32. Choi H, Lee RH, Bazhanov N, Oh JY, Prockop DJ (2011) Anti-inflammatory protein TSG-6 secreted by activated MSCs attenuates zymosan-induced mouse peritonitis by decreasing TLR2/NF-KB signaling in resident macrophages. Blood 118(2):330-8

33. Lee RH, Pulin AA, Seo MJ, Kota DJ, Ylostalo J, Larson BL, Semprun-Prieto L, Delafontaine P, Prockop DJ (2009) Intravenous hMSCS improve myocardial infarction in mice because cells embolized in lung are activated to secrete the anti-inflammatory protein TSG-6. Cell Stem Cell 5(1):54-63

34. Deban L, Russo RC, Sironi M, Moalli F, Scanziani M, Zambelli V, Cuccovillo I, Bastone A, Gobbi M, Valentino S, Doni A, Garlanda C, Danese S, Salvatori G, Sassano M, Evangelista V, Rossi B, Zenaro E, Constantin G, Laudanna C, Bottazzi B, Mantovani A (2010) Regulation of leukocyte recruitment by the long pentraxin PTX3. Nat Immunol 11(4):328-334

35. Wilson JG, Liu KD, Zhuo H, Caballero L, McMillan M, Fang X, Cosgrove K, Vojnik R, Calfee CS, Lee JW, Rogers AJ, Levitt J, Wiener-Kronish J, Bajwa EK, Leavitt A, McKenna D, Thompson BT, Matthay MA (2015) Mesenchymal stem (stromal) cells for treatment of ARDS: a phase 1 clinical trial. Lancet Respir Med 3(1):24-32

36. Chiarini M, Sabelli C, Melotti P, Garlanda C, Savoldi G, Mazza C, Padoan R, Plebani A, Mantovani A, Notarangelo LD, Assael BM, Badolato R (2010) PTX3 genetic variations affect the risk of Pseudomonas aeruginosa airway colonization in cystic fibrosis patients. Genes Immun 11(8):665-670

37. Cunha C, Aversa F, Lacerda JF, Busca A, Kurzai O, Grube M, Löffler J, Maertens JA, Bell AS, Inforzato A, Barbati E, Almeida B, Santos e Sousa P, Barbui A, Potenza L, Caira M, Rodrigues F, Salvatori G, Pagano L, Luppi M, Mantovani A, Velardi A, Romani L, Carvalho A (2014) Genetic PTX3 deficiency and aspergillosis in stem-cell transplantation. N Engl J Med 370(5):421-432

38. Shelburne SA, Ajami NJ, Chibucos MC, Beird HC, Tarrand J, Galloway-Peña J, Albert N, Chemaly RF, Ghantoji SS, Marsh L, Pemmaraju N, Andreeff M, Shpall EJ, Wargo JA, Rezvani K, Alousi A, Bruno VM, Futreal PA, Petrosino JF, Kontoyiannis DP (2015) Implementation of a pan-genomic approach to investigate holobiont-infecting microbe interaction: a case report of a leukemic patient with invasive mucormycosis. PLoS One 10(11):e0139851

\section{Submit your manuscript to a SpringerOpen ${ }^{\circ}$ journal and benefit from:}

- Convenient online submission

- Rigorous peer review

- Immediate publication on acceptance

- Open access: articles freely available online

- High visibility within the field

- Retaining the copyright to your article

Submit your next manuscript at $\boldsymbol{s p r i n g e r o p e n . c o m ~}$ 